\title{
Merging Interface Schemas on the Deep Web via Clustering Aggregation
}

\author{
Wensheng $\mathrm{Wu}$, AnHai Doan \\ University of Illinois, Urbana, USA
}

\author{
Clement $\mathrm{Yu}$ \\ University of Illinois, Chicago, USA
}

\begin{abstract}
We consider the problem of integrating a large number of interface schemas over the Deep Web. The scale of the problem and the diversity of the sources present serious challenges to the conventional manual or rule-based approaches to schema integration. To address these challenges, we propose a novel formulation of schema integration as an optimization problem, with the objective of maximally satisfying the constraints given by individual schemas. Since the optimization problem can be shown to be NP-complete, we develop a novel approximation algorithm LMax, which builds the unified schema via recursive applications of clustering aggregation. We further extend LMax to handle the irregularities frequently occurring among the interface schemas. Extensive evaluation on realworld data sets shows the effectiveness of our approach.
\end{abstract}

\section{Introduction}

The Deep Web consists of a large number of Web databases whose contents are hidden behind their query interfaces. Virtual data integration over Deep Web sources is an emerging research problem which has received great attention (e.g. $[2,4,5,6])$. The challenges of the problem arise largely from two aspects: (1) scale: there are typically a large number of Web databases in any domain of interest; and (2) diversity: these databases often vary greatly in their structure, coverage, vocabulary, and querying capabilities.

As an important step towards the integration of Web databases, we consider the problem of integrating their query interfaces. Query interface to a Web database is typically structured, containing a set of query attributes which are grouped and ordered based on their relative semantics. The structure of the interface can be naturally represented with a hierarchical schema such as ordered tree [6]. To illustrate, Figure 1(a) shows a query interface to an airfare database and Figure 1(b) shows its schema as an ordered tree.

The integration of interface schemas is typically accomplished in two steps: schema matching, which identifies semantic correspondences among interface attributes; and schema merging, which constructs a unified schema given the discovered mappings of the attributes. Such a unified schema should encompass all unique attributes over the given set of interfaces, and should be structurally and semantically well-formed. For example, Figure 1(c) shows schema $S_{v}$ for a different airfare query interface, where attribute marked with $x^{\prime}$ matches with attribute $x$ in the schema $S_{u}$ of Figure 1(b). Figure 1(d) shows a unified schema which integrates the schemas $S_{u}$ and $S_{v}$.

While interface schema matching has been well studied $[4,5,6]$, the problem of merging interface schemas has received relatively little attention. As discussed above, the interface schemas are greatly diversified due to the autonomous nature of the sources. As a result, structural conflicts, as exemplified by the fact that different interfaces may represent a different set of attributes and may organize the attributes in quite different ways, are prevalent over the interfaces. For example, while schema $S_{u}$ arranges the attributes by location and date, schema $S_{v}$ groups them on departure and returning. The resolution of structural conflicts over a large number of interface schemas thus poses serious challenges that call for a novel scalable solution.

In this paper, we present a first systematic study on the problem of merging a large number of interface schemas. We propose a novel formulation of schema integration as an optimization problem (Section 2), where each interface schema in essence expresses certain constraints on the unified schema and the goal is to construct a unified schema such that these constraints are maximally satisfied. Since the optimization problem can be shown to be NP-complete, we propose a novel approximation algorithm LMax, based on recursive applications of clustering aggregation [3] (Section 3). We further extend LMax to cope with the irregularities prevalent over interface schemas (Section 4). Finally, we present the experimental results (Section 5). The extension to LMax for producing ordered schemas is given in the full version of the paper.

\section{Schema Integration as an Optimization Problem}

First, we formally define interface schemas, constraints, and the schema integration problem.

Definition 1: (Interface schema) We view each interface schema as an ordered tree of elements. Each leaf element corresponds to an attribute on the interface. Each internal 


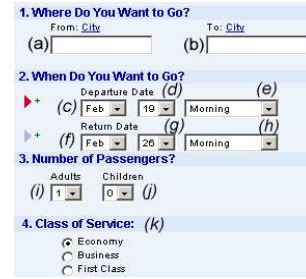

(a) An airfare query interface $Q$

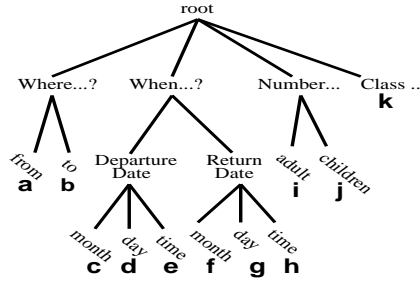

(b) $S_{u}$ : the schema of $Q$

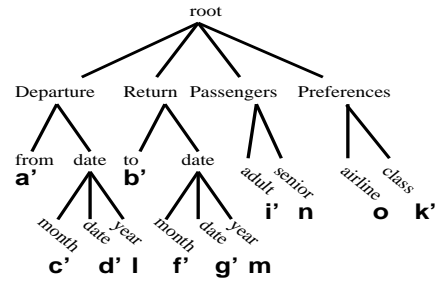

(c) $S_{v}$ : the schema of a different airfare interface

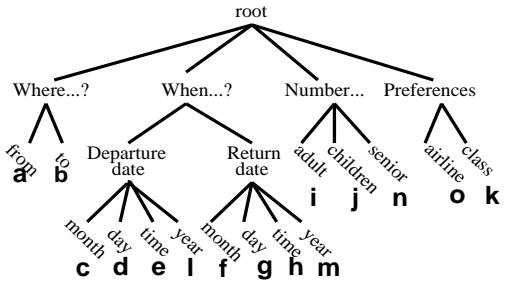

(d) A schema unifying $S_{u}$ and $S_{v}$

FIGURE 1: Examples of query interface, schema, and unified schema

element has an ordered set $s(|s|>1)$ of sub-elements, each of which may be either a leaf element or an internal element. Sub-elements are ordered by the sequence their corresponding attributes (if leaf elements) or groups of attributes (if internal elements) appear on the interface.

For example, Figure 1(b) shows the schema $S_{u}$ for the interface in Figure 1(a). Elements in the schema are annotated with labels from the interface. In the following, we will also use parenthesis notation to represent the schemas. For example, schema $S_{u}$ may be represented as $((a, b)((c$, $d, e)(f, g, h))(i, j) k)$.

As discussed earlier, each of the interface schemas to be integrated essentially expresses certain preferences over the unified schema. These preferences can be encoded in two types of constraints: (a) structural constraints, which are expressed over the ancestor-descendant relationships on the lowest common ancestor (LCA) of attributes and thus restrict the structure of the unified schema; and (b) precedence constraints, which are expressed over the sequence of attributes and thus restrict the ordering of the elements in the unified schema. We now formally define these constraints.

Definition 2: (Structural constraint) Consider a schema $S$ and denote the lowest common ancestor of two attributes $x$ and $y$ in $S$ as $\operatorname{LCA}(x, y)$. Consider three attributes $x, y$ and $z$ in $S$. We say that there exists a structural constraint in form of $(x, y) z$ from $S$, if $\operatorname{LCA}(x, y)<\operatorname{LCA}(x, z)$ and $\operatorname{LCA}(x, y)<\operatorname{LCA}(y, z)$, where $n_{1}<n_{2}$ denotes that element $n_{1}$ is a proper descendant of element $n_{2}$.

Example 1: $(a, b) c$ is a structural constraint from the schema $S_{u}$. Intuitively, it indicates, according to $S_{u}$, attributes $a$ and $b$ (both on the location of flight) are more closely related than either to $c$ (which is on the date of flight).

Given a set of structural constraints without conflicts, [1] gives a polynomial time algorithm to construct a tree which satisfies all the given constraints. But when there are conflicts in the given structural constraints, the algorithm in [1] can not be applied. In our integration problem, conflicts are prevalent. For example, $\left(a^{\prime}, c^{\prime}\right) b^{\prime}$ from $S_{v}$ conflicts with $(a, b) c$ from $S_{u}$. In general, constraint $(x, y) z$ conflicts with $(x, z) y$ and $(y, z) x$. Given two conflicting constraints, it is impossible to find a unified schema which satisfies both constraints.

Definition 3: (Precedence constraint) Consider a schema $S$ and a sequence of attributes, denoted as $q_{s}$, obtained from a pre-order traversal of $S$. We say that there exists a precedence constraint between two attributes $x$ and $y$, denoted as $x \prec y$, from the schema $S$, if $x$ appears before $y$ in $q_{s}$.

Example 2: The sequence $q_{s_{u}}$ for schema $S_{u}$ is $<a, b, c, d, e, f, g, h, i, j, k>$. As such, $a \prec b, a \prec d$ are two precedence constraints from $S_{u}$.

Based on the above definitions, we cast the integration problem as an optimization problem:

Integration problem OPT: Given a set of interface schemas $\mathcal{S}$ with a set of distinct attributes $A$, find a unified schema $G$ such that (1) $G$ 's leaf elements are attributes in $A$; (2) the number of structural constraints from the schemas in $\mathcal{S}$, which are satisfied by $G$, is maximized; and (3) the number of precedence constraints from the schemas in $\mathcal{S}$, which are satisfied by $G$, is maximized.

\section{Approximating OPT via Clustering Aggre- gation}

It is not difficult to prove that OPT is NP-complete. This section presents the algorithm LMax which gives an approximate solution to OPT. Essentially, LMax views the construction of a unified schema as a process of forming recursive partitions over a set of attributes such that structural constraints from the interface schemas are satisfied as much as possible. To illustrate, consider integrating schemas $S_{u}$ and $S_{v}$. To start with, LMax is given a set $A$ of 15 unique attributes (numbered $a-O$ ) over the two schemas. At the first iteration, LMax will create a root node $r$, form a partition over the attributes in $A$, and then create a list of children for $r$, each corresponding to a cluster in the partition. The same process is then recursively applied to each child of $r$, given the attributes in the cluster associated with that child.

We first introduce several necessary concepts.

Definition 4: (Cluster, maximum cluster, and clustering) Consider a schema $S$ which contains a set of attributes 


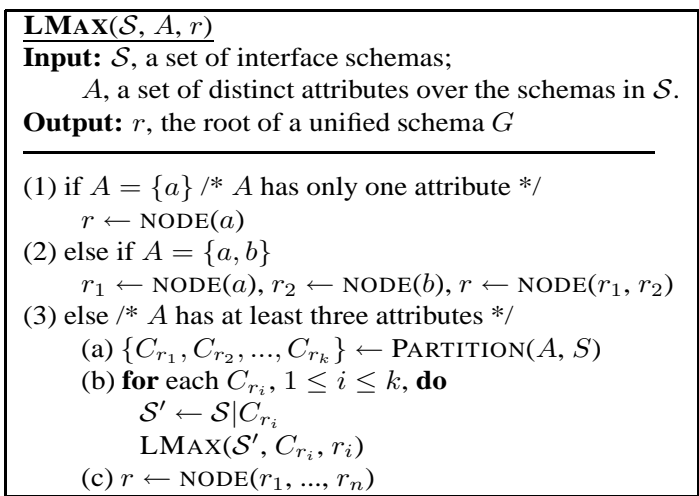

FIGURE 2: The LMax algorithm

$A$. For each node $n$ in $S$, we define a cluster $C_{n}$ as the set of attributes (i.e. leaf elements) in the sub-tree rooted at $n$. A cluster $C$ is a proper cluster if $C \subset A$. A proper cluster is a maximum cluster if it is not a subset of any other proper clusters in $S$. The set of all maximum clusters in $S$ forms a clustering over the attributes in $A$.

Example 3: The maximum clusters in $S_{u}$ are $\{a, b\}$, $\{c, d, e, f, g, h\},\{i, j\},\{k\}$, each associated with a child of the root.

Definition 5: (Restriction) A restriction of a schema $S$ on a set of attributes $X$, denoted as $S \mid X$, is a schema given by: (1) pruning all attributes in $S$ which are not in $X$; (2) pruning the internal node if all its children are pruned; (3) replacing the internal node with only one child by its child.

A restriction of a set of schemas $\mathcal{S}=\left\{S_{1}, \ldots, S_{n}\right\}$ on $X$, denoted as $\mathcal{S} \mid X$, is a set of schemas $\left\{S_{1}\left|X, \ldots, S_{n}\right| X\right\}$.

Example 4: $S_{u} \mid\{a, b, c, d, k\}=((a, b)(c, d) k)$.

Based on the above definitions, LMax algorithm is given in Figure 2. Note that $\operatorname{NOdE}(a)$ creates a leaf node with the attribute $a$; and $\operatorname{NODE}\left(n_{1}, \ldots, n_{k}\right)$ creates an internal node with $n_{i}$ 's as the children.

Given a set of schemas $\mathcal{S}=\left\{S_{1}, S_{2}, \ldots, S_{n}\right\}$, and a set of unique attributes $A$ over the schemas in $\mathcal{S}$, LMax builds a unified schema $G$ as follows. If $A$ has only one attribute $a$, it simply returns $\operatorname{NODE}(a)$ as the root of $G$. If $A$ has only two attributes $a$ and $b, G$ is a tree with two leaves: $\operatorname{NODE}(a)$ and $\operatorname{NODE}(b)$. Otherwise, it first forms a partition $P=\left\{C_{r_{1}}, C_{r_{2}}, \ldots, C_{r_{k}}\right\}$ over the attributes in $A$. Then for each cluster $C_{r_{i}}$, it recursively creates a sub-tree rooted at $r_{i}$ based on a set of restricted schemas $\mathcal{S} \mid C_{r_{i}}$. Finally, it returns $\operatorname{NODE}\left(r_{1}, r_{2}, \ldots, r_{k}\right)$ as the root of $G$.

We now describe the PARTITION function, the key component of the LMax algorithm, in detail.

Partition: Partition $(A, \mathcal{S})$ finds a partition over the attributes in $A$ such that the structural constraints from the schemas in $\mathcal{S}$ are satisfied as much as possible.

Consider a partition $P$ over $A$ where $P=\left\{C_{1}, C_{2}, \ldots\right.$, $\left.C_{k}\right\}$. Consider further a schema $S \in \mathcal{S}$. Suppose that $M=$
$\left\{C_{1}^{\prime}, C_{2}^{\prime}, \ldots, C_{l}^{\prime}\right\}$ is a set of the maximum clusters in $S$. We observe that, to satisfy as many structural constraints from $S$ as possible, $P$ should be such that if two attributes $x, y$ are in the same cluster $C_{i} \in M$, then both $x$ and $y$ should also be placed in the same cluster, say $C_{j} \in P$. Otherwise, all constraints of the form $(x, y) z$ will be violated. On the other hand, having $x, y \in C_{j}$ will satisfy all the constraints of the form $(x, y) z$ for some $z \notin C_{i}$. In other words, we may regard $M$ as a clustering over the attributes in $A$ (possibly with some missing attributes), and a good partition $P$ should be such that it agrees with $M$ on the cluster labels of the attributes.

Denote the set of such clusterings as $\mathcal{M}=\left\{M_{1}, \ldots, M_{n}\right\}$, where $M_{i}$ is the clustering given by the schema $S_{i}$. Since different schemas may give different clusterings, our problem is a problem of clustering aggregation: we seek a partition $P$ such that $P$ maximally agrees with the clusterings in $\mathcal{M}$. Unfortunately, clustering aggregation is also a NPcomplete problem [3]. As such, PARTITION implements an approximation algorithm which can be regarded as a variant of the AGGLOMERATIVE algorithm in [3].

Given a set of attributes $A$ and a set of clusterings $\mathcal{M}=$ $\left\{M_{1}, \ldots, M_{n}\right\}$, PARTITION proceeds as follows. For every pair of attributes $a$ and $b$ in $A$, their potential of being in the same cluster, denoted as $p(a, b)$, is given by the number of clusterings in $\mathcal{M}$ which place $a$ and $b$ in the same cluster, subtracted by the number of clusterings which place $a$ and $b$ in different clusters. PARTITION starts by placing each attribute in $A$ in a cluster by itself. It then repeatedly merges two clusters with the largest potential, where the potential of two clusters is given by the group-average of the potentials of the attributes in the two clusters. The merging process stops when no two clusters have a positive potential.

Example 5: Consider $\mathcal{S}=\left\{S_{u}, S_{v}, S_{w}\right\}$, where $S_{u}$ and $S_{v}$ are schemas in Figure 1(b) and 1(c) respectively, and $S_{w}$ is homogeneous to $S_{u}$ (with the same attributes and structure). Thus, $\mathcal{S}$ contains a set $A$ of 15 unique attributes numbered from $a$ to $o$. The first call to PARTITION with $A$ and $\mathcal{S}$ returns $P=\{\{a, b\},\{c, d, e, f, g, h, l, m\},\{i, j, n\},\{k, o\}\}$. By recursive applications of PARTITION on each cluster in $P$, LMax produces a unified schema shown in Figure 1(d).

\section{Handling Irregular Interface Schemas}

Compared to other types of schemas, e.g., schemas in relational databases, interface schemas are typically much less regular. In particular, we observe that the structure of some interfaces may be implicit in that the attributes may be simply listed one after another without explicit group delimiters. This poses challenges to schema extraction algorithms. As a result, the obtained schema may not fully capture the grouping relationships of attributes on the interface. The irregular schemas may greatly affect the performance of PARTITION which assumes that maximum clus- 
ters of each schema $S$ indicate $S$ 's preferences on dividing attributes into groups.

To address this challenge, consider again a set of schemas $\mathcal{S}$, some of which may be irregular. A key observation is that we can exploit other schemas in $\mathcal{S}$ to identify the irregularities in the irregular schemas, assuming that not every schema in $\mathcal{S}$ is irregular. Specifically, consider a schema $S_{i} \in \mathcal{S}$. We observe that if attributes $a, b \in S_{i}$ appear in different maximum clusters of $S_{i}$, but both appear in the same maximum cluster of some other schema $S_{j} \in \mathcal{S}$, then it is very likely that the grouping relationship between $a$ and $b$ is implicit on the interface of $S_{i}$. Motivated by the above observation, we extend LMax based on the concept of global maximum clusters defined as follows.

Definition 6: (Global maximum cluster) Consider a set of schemas $\mathcal{S}=\left\{S_{1}, \ldots, S_{n}\right\}$, where each schema $S_{i} \in \mathcal{S}$ gives a set of maximum clusters $M_{i}=\left\{C_{i_{1}}, C_{i_{2}}, \ldots, C_{i_{k}}\right\}$. We say that a maximum cluster $C_{i_{j}} \in M_{i}$ is a global maximum cluster if it is not a proper subset of any maximum clusters of any other schemas in $\mathcal{S}$.

We denote the set of global maximum clusters over the schemas in $\mathcal{S}$ as $\mathcal{C}^{\mathcal{S}}=\left\{C_{1}^{S}, C_{2}^{S}, \ldots, C_{m}^{S}\right\}$, and denote the set of unique attributes in the schemas of $\mathcal{S}$ as $A$. (It is important to note that the clusters in $\mathcal{C}^{\mathcal{S}}$ may not form a partition over $A$, due to structural conflicts in the schemas of $\mathcal{S}$.)

Based on the above definition, we modify PARTITION in LMax. Recall that, given a set of attributes $A$ and a set of clusterings $\mathcal{M}=\left\{M_{1}, \ldots, M_{n}\right\}$, where $M_{i}$ is a set of maximum clusters obtained from schema $S_{i} \in \mathcal{S}$, PARTITION forms a partition over $A$ via clustering aggregation.

The modified PARTITION consists of the following steps: (1) obtain $\mathcal{C}^{\mathcal{S}}$, the set of global maximum clusters; (2) transform $\mathcal{M}$ into a new set of clusterings $\mathcal{M}^{\prime}=\left\{M_{1}^{\prime}, \ldots, M_{n}^{\prime}\right\}$, where $M_{i}^{\prime}$ is obtained from $M_{i}$ by combining clusters in $M_{i}$, which are subsets of the same global maximum cluster in $\mathcal{C}^{\mathcal{S}}$, into one cluster; (3) perform the clustering aggregation with $\mathcal{M}^{\prime}$ instead of $\mathcal{M}$.

The LMax algorithm with the new PARTITION is denoted as GMax.

\section{Empirical Evaluation}

We have evaluated both the LMax and GMax algorithms on a real-world data set over varied domains. The goal of the experiments was to examine if the produced unified schemas are semantically well-formed, and to compare the performance of the two algorithms.

For all experiments, we used a data set which contains a total of 100 interface schemas extracted from the query interfaces to Web databases in five domains: airfare, auto, book, job, and real estate, with 20 schemas for each domain. Table 1 gives the statistics of the data set.

\begin{tabular}{|c|r|r|r|r|r|r|r|r|r|r|}
\hline \multirow{2}{*}{ Domain } & \multicolumn{3}{|c|}{ Leaf Nodes } & \multicolumn{3}{c|}{ Internal Nodes } & \multicolumn{3}{c|}{ Depth } \\
\cline { 2 - 11 } & Min & Max & Avg & Min & Max & Avg & Min & Max & Avg \\
\hline Airfare & 5 & 15 & 10.7 & 1 & 7 & 5.1 & 2 & 5 & 3.6 \\
\hline Automobile & 2 & 10 & 5.1 & 1 & 4 & 1.7 & 2 & 3 & 2.4 \\
\hline Book & 2 & 10 & 5.4 & 1 & 2 & 1.3 & 2 & 3 & 2.3 \\
\hline Job & 3 & 7 & 4.6 & 1 & 2 & 1.1 & 2 & 3 & 2.1 \\
\hline Real Estate & 3 & 14 & 6.7 & 1 & 6 & 2.4 & 2 & 4 & 2.7 \\
\hline
\end{tabular}

TABLE 1: Domains and statistics of the data set

An objective measure on the quality of a produced unified schema is to compare the unified schema with the optimal unified schema. But since finding optimal schemas is computationally expensive, we use an alternative measure PerSC, which is the percentage of strong structural constraints from the given set of interface schemas satisfied by the unified schema. A structural constraint $(x, y) z$ is a strong constraint if it appears more often in the given set of interface schemas than its conflicting constraints, i.e., $(x, z) y$ and $(y, z) x$. Intuitively, since conflicting constraints from different schemas can not be satisfied simultaneously, we expect that the optimal schema should satisfy the strong constraints.

\begin{tabular}{|c||c|c|c|c|c||c|}
\hline Alg. & Airfare & Auto & Book & Job & Real Est. & Average \\
\hline \hline LMax & 73.6 & 74.0 & 91.8 & 73.7 & 63.6 & 75.3 \\
\hline GMax & 89.9 & 95.6 & 91.8 & 89.5 & 89.0 & 91.2 \\
\hline
\end{tabular}

TABLE 2: The performance of LMax vs. GMax

Table 2 shows the performance of LMax and GMax on the data set, measured by their PerSC scores. We observe that the PerSC scores of LMax range from $63.6 \%$ in the real estate domain to $91.8 \%$ in the book domain. We further observe that GMax improves the performance significantly over four domains, with a $15.8 \%$ increase in the job domain and as high as $25.4 \%$ increase in the real estate domain. These indicate the prevalence of irregularities in the interface schemas. Overall, the average PreSC score increases from $75.3 \%$ to $91.2 \%$. This indicates the effectiveness of GMax in handling the irregular interface schemas.

\section{References}

[1] A. Aho, Y. Sagiv, T. Szymanski, and J. Ullman. Inferring a tree from lowest common ancestors with an application to the optimization of relational expressions. SIAM, 10(3).

[2] L. Barbosa and J. Freire. Searching for hidden-web databases. In WebDB, 2005.

[3] A. Gionis, H. Mannila, and P. Tsaparas. Clustering aggregation. In ICDE, 2005.

[4] B. He and K. Chang. Statistical schema matching across Web query interfaces. In Proc. of SIGMOD, 2003.

[5] H. He, W. Meng, C. Yu, and Z. Wu. Wise-integrator: an automatic integrator of web search interfaces for e-commerce. In $V L D B, 2003$.

[6] W. Wu, C. Yu, A. Doan, and W. Meng. An interactive clustering-based approach to integrating source query interfaces on the Deep Web. In SIGMOD, 2004. 